\title{
Fast response tunable diode laser spectroscopy for trace gas flux measurements
}

\author{
F.G. Wienhold, H. Fischer \\ Air Chemistry Department, Max Planck Institute for Chemistry, P.O. Box 3060, D-55020 Mainz, Germany \\ G.W. Harris \\ Centre for Atmospheric Chemistry, York University, 4700 Keele St., North York, Ontario M3J 1P3, Canada
}

\begin{abstract}
A fast tunable diode laser spectrometer was applied for measurements of $\mathrm{N}_{2} \mathrm{O}$ emissions from a harvested wheat field located in Sealand, Denmark. The dual-channel instrument uses two tone frequency modulation and signal detection at $11 \mathrm{MHz}$ in conjunction with rapid (1 ms) scanning of the laser. A dichroic beam combiner and a mechanical chopper allow time multiplexing. The absorption signals are recorded and analysed on-line. The combination of a software, current and temperature controlled line locking scheme results in high stability of the instrument. The time response $(200 \mathrm{~ms})$ of the spectrometer is limited by the measurement-cell gas exchange. For eddy correlation measurements, the trace gas mixing ratio and wind data were sampled synchronously at a rate of $10 \mathrm{~Hz}$ using an interrupt-driven algorithm.

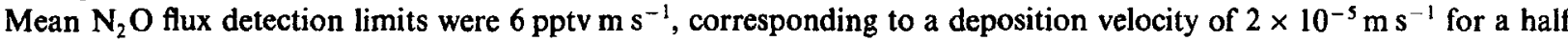
hour measurement time. In addition, concentration gradients were determined from measurements at four different inlet heights at $0.1 \mathrm{ppbv}$ precision in a shared integration time of $30 \mathrm{~min}$. These measurements resulted in a flux detection

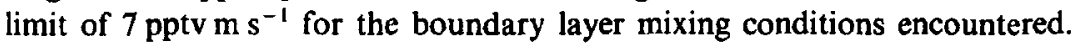

\section{Introduction}

It has been demonstrated in laboratory studies that the performance of tunable diode laser absorption spectrometers (TDLAS) can be improved substantially by the application of high frequency modulation techniques [1]. For field spectrometers used to monitor atmospheric trace gases, the stability of the optical background structure most commonly limits the attainable sensitivity. In this case, high frequency modulation has still the potential to decrease the measurement averaging time, which is desirable for various applications. If, for example, the concentration of an atmospheric trace component and the vertical wind velocity are measured simultaneously at the same location with adequate time resolution, the vertical flux of the trace component at this location can be derived from the covariance of the two measurements (the eddy correlation technique) [2]. Taking into 
account certain chemical and micrometeorological restrictions, this method is capable of quantifying the gas emission or absorption averaged over large areas of the surface [3]. While the eddy correlation technique is well established for the measurement of micrometeorological quantities (e.g. momentum and heat flux) $[4,5]$, its utilization for trace gas flux measurement has been severely limited because of the response speed and precision imposed on the trace gas sensor.

Trace gas emissions or absorptions from soils, although often poorly quantified, are considered to be a significant contribution to the atmospheric trace gas budgets [6]. Eddy correlation flux measurements for source quantification are highly desirable as a complement to the commonly used chamber techniques, compared to which they exhibit two major advantages. Firstly, the surface flux contributions from an area of $10^{4}$ to $10^{5} \mathrm{~m}^{2}$ size are integrated, which is important for a representative measurement as the small scale spatial flux variability is usually large. Secondly, the flux measurement does not change the environmental conditions controlling the emission or absorption process. These features are common to most micrometeorological methods for flux quantification, some of which do not require a fast response trace gas sensor (e.g. gradient techniques or conditional sampling). In a practical field experiment however, the micrometeorological assumptions on which these approaches rely are usually violated to a certain extent leading to additional systematical errors which are absent in the "direct" eddy correlation measurement.

We describe a fast response, two tone frequency modulation (TTFM) TDLAS and its application

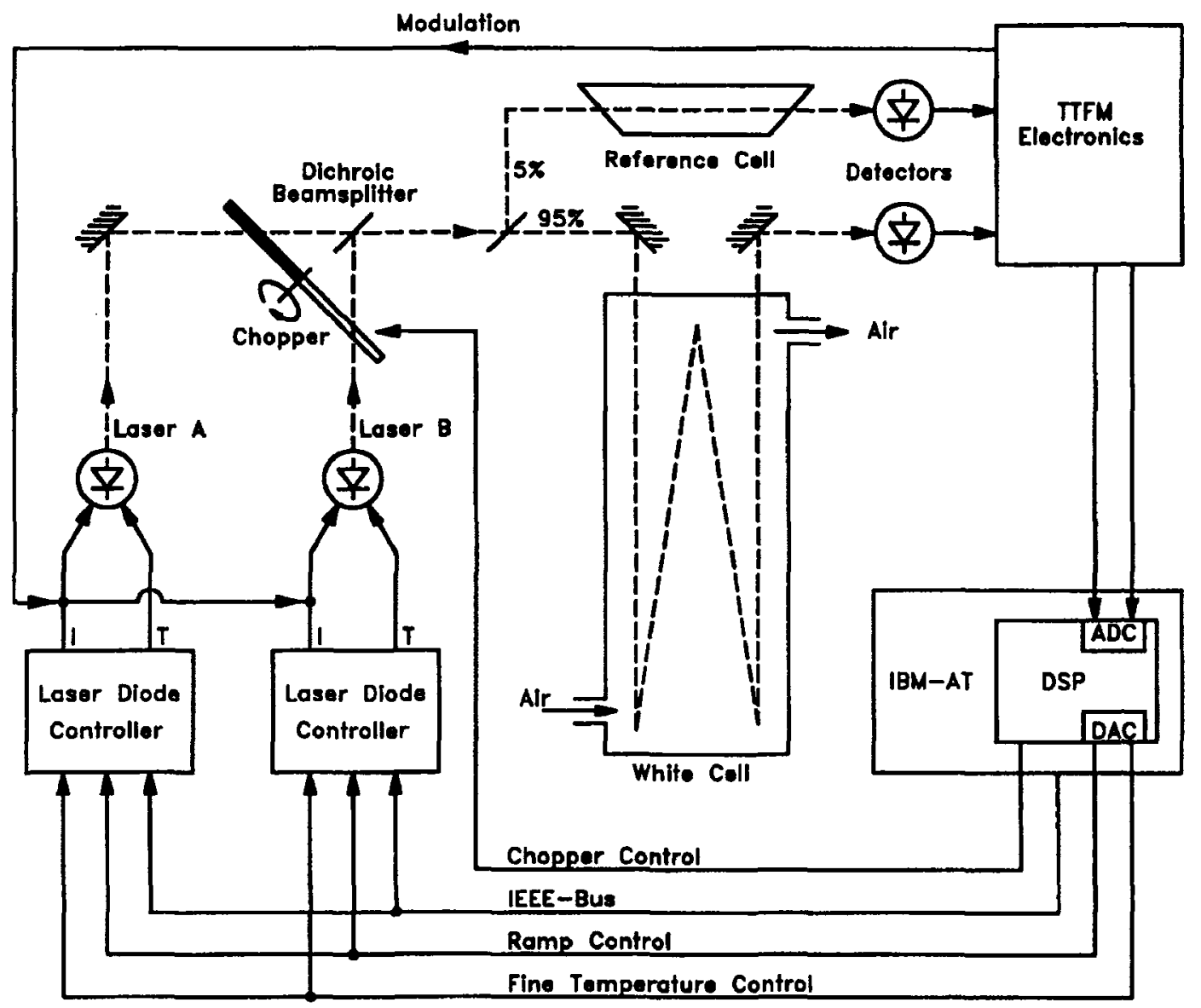

Fig. 1. Schematic of the TDLAS. 
for flux measurements of nitrous oxide using the eddy correlation technique. The experiment was carried out as part of an international field campaign comparing different methods to quantify nitrous oxide soil emissions which took place on a harvested wheat field located in northwest Sealand (Denmark) during Autumn 1993.

\section{The TTFM spectrometer}

The spectrometer measures the concentration of a specific trace gas by quantifying the attenuation of infrared light caused by the excitation of an individual rotational-vibrational molecular transition. The intensity of the absorption line is repetitively recorded by scanning the laser emission using diode current variation. The instrument uses two lead-salt diode lasers to monitor two different trace gases simultaneously, as illustrated in Fig. 1. The beams emerging from the diodes are collimated by $90^{\circ}$ OAPs, combined by a dichroic beam splitter and selected alternatively by a rotating chopper. The main fraction of the chosen laser light traverses an absorption cell in which the beam is folded by a mirror arrangement (double corner cube White cell) [7] to increase the absorption path and hence instrument sensitivity. Sample air is drawn through the cell at a high exchange rate, which controls the instrumental time response. The light leaving from the cell is collected by a high bandwidth detector ( $20 \mathrm{MHz})$. In parallel, a small light fraction is coupled through a single-pass reference cell containing the target trace gases at high concentration onto a second detector. The reference cell absorption is used to monitor the wavelength region covered by the laser scans relative to the absorption feature. Deviations are compensated during signal averaging, and by readjusting the laser current and temperature via the laser diode controllers (line locking). A two tone frequency modulation (TTFM) scheme reduces noise and background in the absorption spectra and allows fast scanning of the laser. A digital signal processor (DSP) hosted by an IBM-AT compatible computer records and analyses the spectral signals in real time, which means that the sampling time equals the spectral averaging
Table 1

Spectrometer properties

\begin{tabular}{ll}
\hline Characteristic & Value \\
\hline Dimensions $(W \times H \times D)$ & $95 \times 145 \times 60 \mathrm{~cm}^{3}$ \\
Optical cell path length & $38.7 \mathrm{~m}$ \\
Optical cell volume & 2.71 \\
Modulation frequencies & $300 \pm 5.5 \mathrm{MHz}$ \\
Detection frequency & $11 \mathrm{MHz}$ \\
Chopper interval & $25 \mathrm{~ms}$ \\
Scan time & $1 \mathrm{~ms}$ \\
Resolution of the spectrum & 100 channels \\
\hline
\end{tabular}

time. Simultaneously, the DSP controls the chopper phase and generates the laser current ramp signal. The specifications of the spectrometer are summarized in Table 1.

Spectrometer performance was investigated using a one hour data set on the nitrous oxide mixing ratio at a sampling rate of $10 \mathrm{~Hz}$ in motionless laboratory air. This primary data set was smoothed by a numerical low pass filter with variable integration time. Means and standard deviations $(2 \sigma)$ of the resulting data sets are displayed in Fig. 2 (left) as a function of the filter time constant. The 2 ppbv standard deviation of the primary data set falls below the $0.1 \mathrm{ppbv}$ level as the averaging time reaches $1000 \mathrm{~s}$. A least squares fit of a straight line to the data indicates a spectrometer drift smaller than $0.05 \mathrm{ppbv} / \mathrm{h}$. Spectrometer noise and drift contributions are revealed in more detail by analysing the power spectral density of the primary data set obtained by Fourier transformation (Fig. 2 right). In the double-logarithmic representation, $0 \mathrm{~dB}$ correspond to the tropospheric nitrous oxide mixing ratio of $310 \mathrm{ppbv}$. Since the power spectral density is related to the square of the parent quantity, the $-70 \mathrm{~dB}$ level is equivalent to $0.1 \mathrm{ppbv}$ mixing ratio. At the low frequency end of the graph the drift contributions are reflected by the departure of the power spectral density from the dashed line which indicates a white noise behaviour. The low spectrometer drift is achieved through the combined software, current and temperature line-locking algorithm, each element of which uses different time scales [8]. This spectrometer performance was also reproduced under field conditions $[9,10]$. The spectrometer features relevant for trace gas flux measurements are assembled in Table 2. 

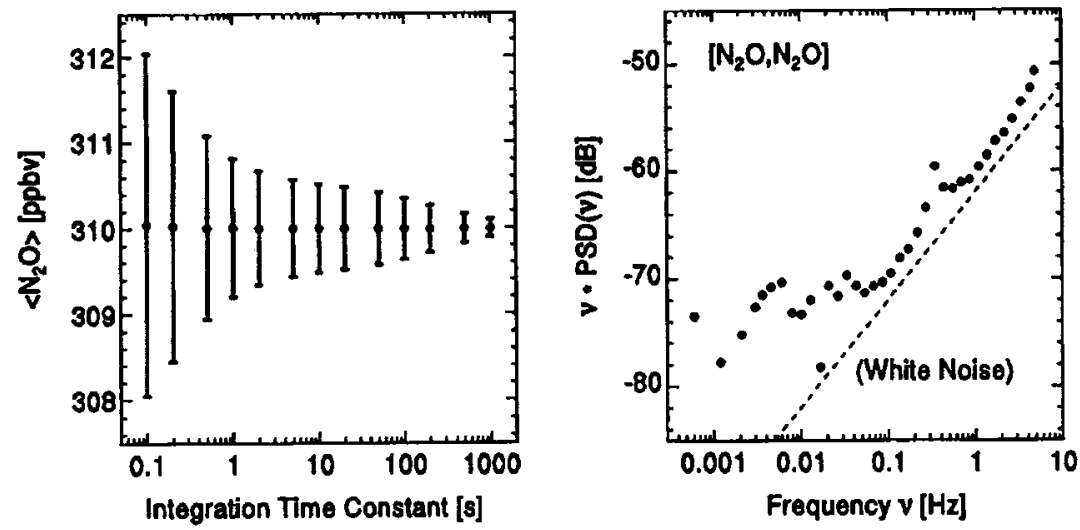

Fig. 2. Noise and drift analysis.

Table 2

Spectrometer performance

\begin{tabular}{ll}
\hline Characteristic & Value \\
\hline On-line data reduction & $\geqslant 20 \mathrm{~ms}$ \\
Optical cell gas exchange & $200 \mathrm{~ms}$ \\
Precision (0.1 s averaging time) & $2 \mathrm{ppbv}$ \\
Precision (16 min averaging time) & $0.1 \mathrm{ppbv}$ \\
Drift & $<0.05 \mathrm{ppbv} / \mathrm{h}$ \\
\hline
\end{tabular}

\section{Application to nitrous oxide flux measurements}

A full description of the Denmark field campaign is given elsewhere [11]. The arrangement for nitrous oxide flux measurements using the TDLAS is shown in Fig. 3. For eddy correlation, the air was sampled close to a ultrasonic anemometer which supplied three-dimensional wind and temperature

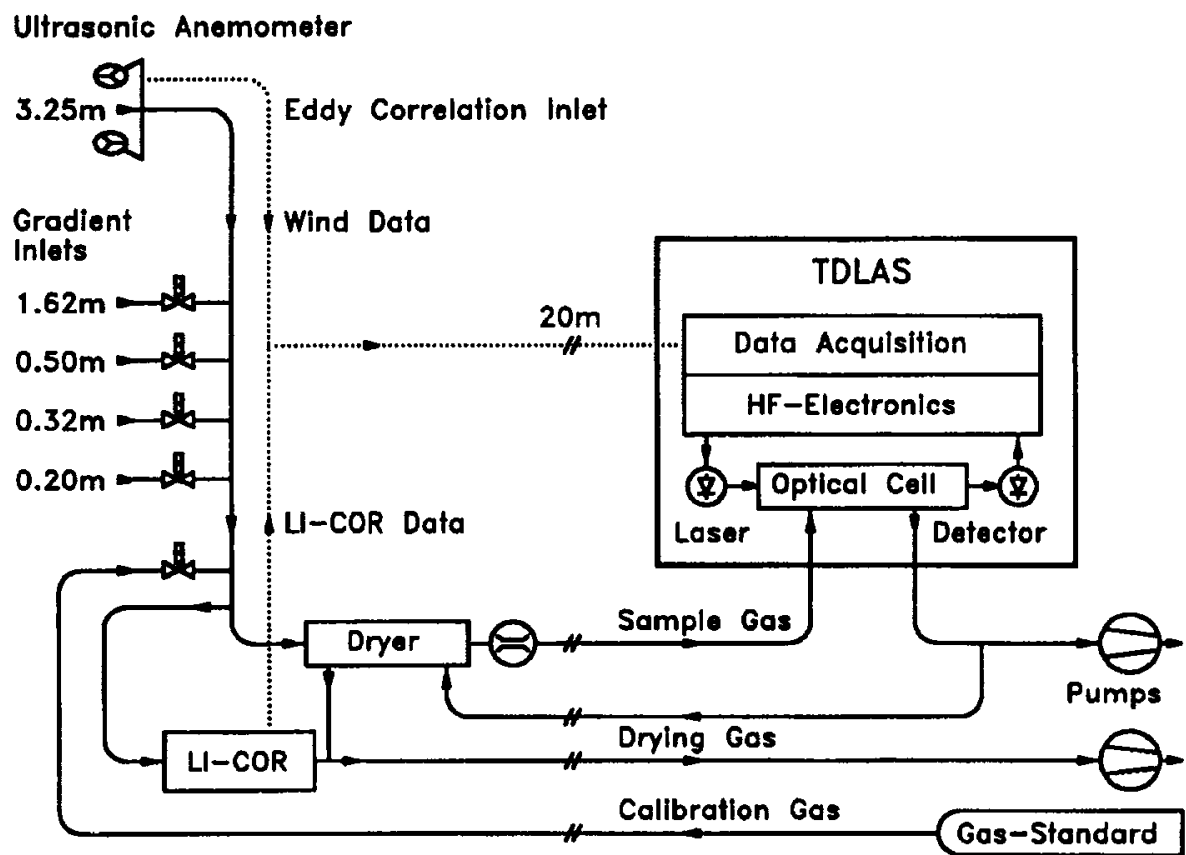

Fig. 3. Experimental setup. 


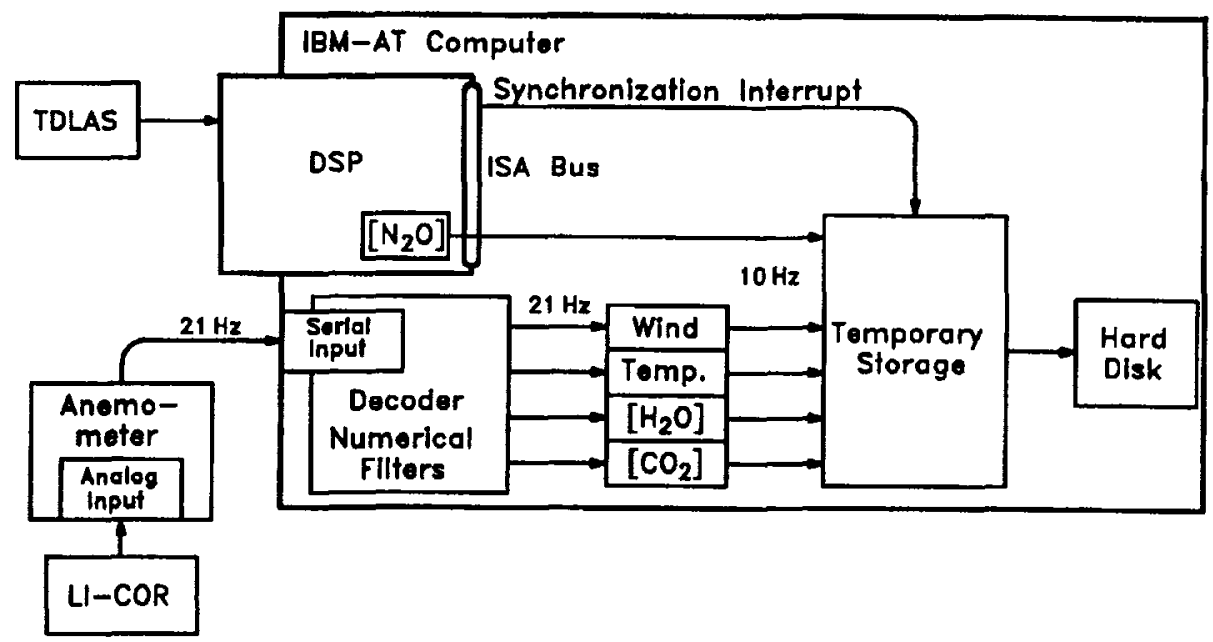

Fig. 4. Data acquisition for eddy correlation measurements.

data. Water vapour and carbon dioxide concentrations of the gas were determined by a non-dispersive infrared analyser (LI-COR). The major fraction of the sample was dried and equilibrated to constant temperature (to avoid the necessity of density corrections [12]) while being conducted to the mobile laboratory containing the TDLAS. A pumping rate of $700 \mathrm{l} / \mathrm{min}$ resulted in a gas exchange time of $0.2 \mathrm{~s}$ within the optical cell which equalled the instrumental time response, as verified experimentally. Using a sampling time of $0.1 \mathrm{~s}$ the measurement cell gas exchange served as a "natural" anti-aliasing low pass filter. At half hour intervals, the TDLAS was calibrated using standard gas.

As indicated in Fig. 4, wind, temperature and concentration values were sampled synchronously, as required for the covariance analysis. The data acquisition timing was controlled by the TDLAS DSP computer. Water vapour and $\mathrm{CO}_{2}$ concentrations were read by analog inputs from the anemometer and transmitted at a $21 \mathrm{~Hz}$ rate together with wind and temperature values to the serial port of the AT spectrometer computer. Numerical low pass filters adapted the bandwidth to the lower sampling rate of the TDLAS. Each time a $\mathrm{N}_{2} \mathrm{O}$ concentration was established by the DSP programme (i.e., every $0.1 \mathrm{~s}$ ) an interrupt was sent to the AT to transfer the whole data sample into the AT memory. Time series of $2^{14}$ samples resulting in a $27 \mathrm{~min}$ measurement interval were collected and subsequently stored on the computer hard disk. The bandwidth of the eddy correlation measurement was thus $6.1 \times 10^{-4} \mathrm{~Hz}$ to $5 \mathrm{~Hz}$ according to the sampling theorem.

Additional inlets at four different heights were used for concentration gradient measurements (see Fig. 3) carried out occasionally for comparison with other equipment available on the site. In this case the sampling time was set to $1 \mathrm{~s}$, and the four intake heights were selected successively by switching the gradient inlets at $20 \mathrm{~s}$ intervals and collecting data for 30 min periods between instrument calibrations. To deduce the $\mathrm{N}_{2} \mathrm{O}$ flux from the measured concentration gradient, the mean atmospheric transfer coefficient during the measurement interval was evaluated from the momentum and heat fluxes [13].

\section{Results}

The individual eddy correlation data time series were analysed off line to obtain the nitrous oxide flux, together with those of the other measured quantities (carbon dioxide, momentum, sensible and latent heat). Cospectra and covariances were evaluated by fast Fourier transformation. Fig. 5 shows the result of the data analysis for the case of the $\mathrm{N}_{2} \mathrm{O}$ flux. On the left, the power spectral density of the $\mathrm{N}_{2} \mathrm{O}$ concentration measured in the 

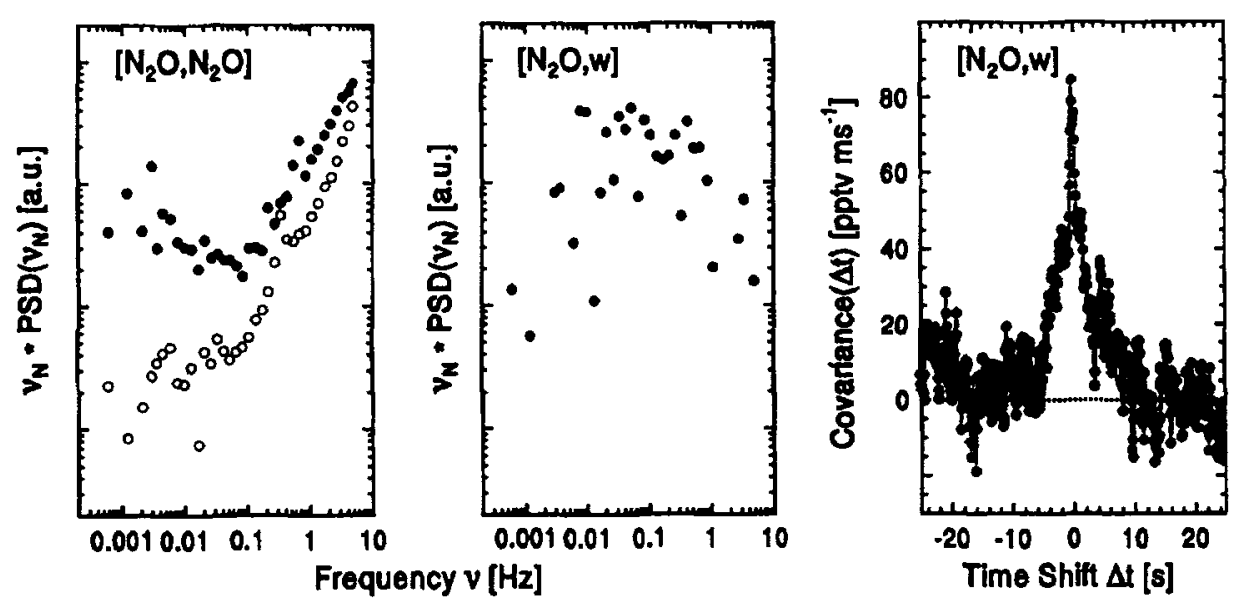

Fig. S. Eddy correlation measurement result.

presence of a vertical flux (full circles) is compared to the data presented in Fig. 3, indicated as empty circles. The concentration fluctuations caused by the flux are clearly evident in the low frequency region, while the high frequency contributions appear to be masked by the instruments $1 / f$ noise. However, since the noise fluctuations are not correlated to the vertical wind, the flux contributions can be distinguished from instrumental noise (and drift) by considering the cospectrum of $\mathrm{N}_{2} \mathrm{O}$ and the vertical wind $w$, shown in the middle of Fig. 5 . The filter effect of the eddy correlation measurement can be interpreted as that of phase sensitive detection driven by a stochastical reference. Inverse Fourier transform of the cospectrum results in the covariance function (right hand graph in Fig. 5). The flux value was computed from the central peak of the covariance function, while the off-peak noise level served as an estimate for defining the flux detection limit.

The other supporting fluxes were evaluated in the same way. They were analysed for information on atmospheric stability and thus transport characteristics of the boundary layer. In addition, the investigation of the energy balance between the fluxes of sensible and latent heat, as determined by eddy correlation, and those of net radiation and soil heat, measured independently by other devices [13], revealed that the bandwidth interval defined by the sampling rate and measurement time was adequate to cover the flux contributions. System-

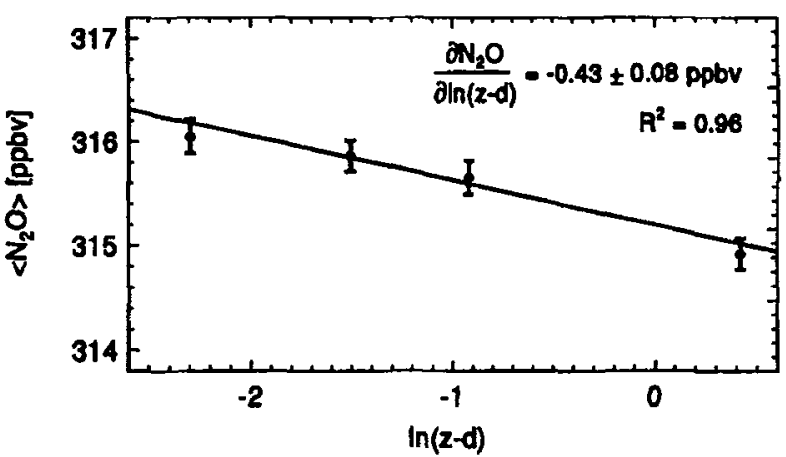

Fig. 6. $\mathrm{N}_{2} \mathrm{O}$ concentration gradient measurement.

atic errors were below $5 \%$, and statistical errors due to storage and advection were less than $18 \%$.

Fig. 6 shows a concentration gradient measured with the TDLAS. The data represent a total half hour measurement time equally distributed between the four intake heights. The low spectrometer drift allows the determination of a $\mathrm{N}_{2} \mathrm{O}$ concentration gradient with a precision of $80 \mathrm{pptv}$ at the ambient level of $310 \mathrm{ppbv}$. In applying the gradient technique for $\mathrm{N}_{2} \mathrm{O}$ flux measurements, the uncertainty associated with the estimation of the atmospheric transfer coefficients [14] was of similar magnitude as the error in the gradient determination for the conditions experienced during the campaign. Table 3 gives an overview of the error contributions and the total error estimate for both flux measurement methods. The detection flux limits quoted are mean values for the whole experiment period. The 
Table 3

Errors and detection limits of the $\mathrm{N}_{2} \mathrm{O}$ flux measurements

\begin{tabular}{lll}
\hline Method & Error source & Error \\
\hline Gradient method & Mixing ratio determination & $16 \%$ \\
& Micromet assumptions & 10 to $30 \%$ \\
Total error & & $26 \%$ \\
Detection limit & & $7 \mathrm{pptv} \mathrm{m} \mathrm{s}^{-1}$ \\
Eddy correlation & Covariance measurement & $10 \%$ \\
& Bandwidth coverage (systematic) & $<5 \%$ \\
Total error & Bandwidth coverage (statistical) & $<18 \%$ \\
Detection limit & & $21 \%$ \\
\hline
\end{tabular}

aDeduced from energy balance

$\mathrm{N}_{2} \mathrm{O}$ emissions encountered during that time lay

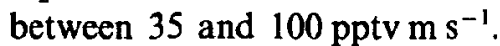

\section{Summary}

The sensitivity and time resolution attainable with high-frequency modulated tunable diode laser absorption spectroscopy encourages its use for correlation measurements. The application of eddy correlation for trace gas flux determination is attractive because it avoids interference with the system studied and integrates the emitted or absorbed flux over convenient areas. In addition, it quantifies the flux direction without requiring additional micrometeorological measurements or assumptions. The instrument described, together with the corresponding data recording and analysing algorithm has proven capable for eddy correlation flux measurements of $\mathrm{N}_{2} \mathrm{O}$ during a field campaign. Considering the tropospheric $\mathrm{N}_{2} \mathrm{O}$ mixing ratio of $310 \mathrm{ppbv}$, the detection limit of

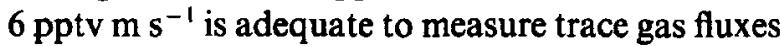
with deposition velocities down to $2 \times 10^{-5} \mathrm{~m} \mathrm{~s}^{-1}$ using $30 \mathrm{~min}$ averaging time. Due to the high ambient mixing ratio, an absorption with only one tenth line strength of the strongest lines in the $\mathrm{N}_{2} \mathrm{O}$ band at $2200 \mathrm{~cm}^{-1}$ was sufficient to yield an optical density of $1 \%$. Taking into account that stronger lines could be used for species with lower background concentrations (e.g. NO, OCS), the flux detection limits could easily be improved for these molecules.

\section{Acknowledgement}

This work was supported in part by funding from the European Community Science and Technology for Environmental Protection program (EC contract STEP CT90-0028) and the Max Planck Society. We thank Horst Frahm and Michael Welling for assistance in the field measurement components.

\section{References}

[1] D.E. Cooper and R.E. Warren, J. Opt. Soc. Am. B 4 (1987) 470.

[2] R.T. McMillen, Boundary Layer Meteorol. (1988) 213.

[3] D. Fowler and J.H. Duyzer, Exchange of Trace Gases Between Terrestrial Ecosystems and the Atmosphere, eds. M.O. Andreae and D.S. Schimel (John Wiley, New York, 1989) pp. 189-207.

[4] A.J. Dyer and F.J. Maher, J. Appl. Meteorol. 4 (1965) 622.

[5] A.J. Dyer, B.B. Hicks and K.M. King, J. Appl. Meteorol. 6 (1967) 408.

[6] J.T. Houghton, B.A. Callander and S.K. Varney, Climate Change 1992: The Supplementary Report to the IPCC Scientific Assessment, Intergovernmental Panel on Climate Change (Cambridge University Press, Cambridge, 1992).

[7] J.U. White, J. Opt. Soc. Am. 66 (1976) 411.

[8] F.G. Wienhold, T. Zenker and G.W. Harris, A dual channel two tone frequency modulation tunable diode laser spectrometer for ground-based and airborne trace gas measurements, in: Tunable Diode Laser Spectroscopy, Lidar and DIAL Techniques for Environmental and Industrial Measurements, eds. A. Fried, D.K. Killinger and H.I. Schiff, Proc. Spie 2112 (1994) 31.

[9] F.G. Wienhold, H. Frahm and G.W. Harris, J. Geophys. Res. 99 (D8) (1994) 16557. 
[10] F.G. Wienhold, M. Welling and G.W. Harris, submitted to Atmos. Environ. 29 (17) (1995) 2219.

[11] S. Christensen, P. Ambus, J.R.M. Arah, H. Clayton, B. Galle, D.W.T. Griffith, K.J. Hargreaves, L. Klemedtsson, A.M. Lind, M. Maag, A. Scott, U. Skiba, K.A. Smith, M. Welling and F.G. Wienhold, submitted to Atmos. Environ (1995).
[12] E.K. Webb, G.I. Pearman, and R. Leuning, Q. J. R. Meteorol. Soc. 106 (1980) 85.

[13] K.J. Hargreaves, F.G. Wienhold, L. Klemedtsson, J.R.M. Arah, I.J. Beverland, D. Fowler, B. Galle, D.W.T. Griffith, U. Skiba, K.A. Smith, M. Welling and G.W. Harris, submitted to Atmos. Environ. (1995).

[14] J.A. Businger, J. Climate Appl. Meteorol. 25 (1986) 1100. 